\title{
Neurobehavioral dimensions of Prader Willi \\ Syndrome: Relationships between sleep disturbance and psychotic experiences
}

\section{Zizhao Zhang}

UCLA: University of California Los Angeles

Ariana Vajdi

UCLA: University of California Los Angeles

Leila Kushan-Wells

UCLA: University of California Los Angeles

Zhengyi Sissi Huang

UCLA: University of California Los Angeles

Laura Pacheco-Hansen

UCLA: University of California Los Angeles

Elizabeth Roof

Vanderbilt University

Anthony Holland

University of Cambridge

Allison M Port

University of Pennsylvania

Ruben C. Gur

University of Pennsylvania

Carrie E Bearden ( $\square$ CBearden@mednet.ucla.edu )

UCLA: University of California Los Angeles https://orcid.org/0000-0002-8516-923X

Research

Keywords: Prader Willi Syndrome, psychosis, sleep, social cognition

Posted Date: December 21st, 2020

DOI: https://doi.org/10.21203/rs.3.rs-131462/v1

License: (c) (1) This work is licensed under a Creative Commons Attribution 4.0 International License.

Read Full License 


\section{Abstract}

Background: Prader Willi Syndrome (PWS) is a genetic disorder caused by the absence of expression of the paternal copies of maternally imprinted gene(s) located at 15q11-q13. While the physical and medical characteristics of PWS, including short stature, hyperphagia and endocrine dysfunction are wellcharacterized, systematic investigation of the long-recognized psychiatric manifestations has been recent.

Methods: Here, we report on the first remote (web-based) assessment of neurobehavioral traits, including psychotic-like experiences (Prodromal Questionnaire-Brief Version; PQ-B) and sleep behavior (Pittsburgh Sleep Quality Index), in a cohort of 127 participants with PWS, of whom $48 \%$ had a paternal deletion, $36 \%$ uniparental disomy, 2.4\% an imprinting mutation and 13\% unknown mutation (mean age 19.2 years $+/-8.4$ years; $55.2 \%$ female). We aimed to identify the most informative variables that contribute to psychosis risk symptoms. Multiple domains of cognition (accuracy and speed) were also assessed in a subset of PWS participants ( $\mathrm{N}=39)$ using the Penn Computerized Neurocognitive Battery (Penn-CNB).

Results: Sleep disturbance, particularly the reasons for sleep difficulty, was most strongly associated with distress related to psychotic-like symptoms. Regarding cognition, individuals with PWS showed the most prominent deficits in accuracy on measures of social cognition involving faces, namely Face Memory, Age Differentiation and Emotion Recognition, and greatest slowing on measures of Attention and Emotion Recognition. However, there were no significant differences in psychotic-like experiences or cognitive performance as a function of PWS genetic subtype.

Conclusion: Findings indicate that self/parent-reported neurobehavioral symptoms and cognition can be assessed remotely in individuals with PWS, which has implications for future large-scale investigations of rare neurogenetic disorders.

Trial registration: Not applicable.

\section{Introduction}

Prader Willi Syndrome (PWS) is a genetic disorder caused by the absence of expression of the paternal copies of maternally imprinted gene(s) genes located at 15q11-q13 (Webb et al., 2002). About $70 \%$ of people with PWS have a deletion, $25 \%$ are caused by maternal uniparental disomy (mUPD) and a minority are due to imprinting defects or translocation in the PWS region. PWS is associated with a number of physical and medical conditions including hypotonia, short stature, endocrine dysfunction and childhoodonset hyperphagia (Cassidy et al., 2012). While these aspects of the disorder are now well-characterized, the psychiatric manifestations have only recently begun to be systematically investigated. The neurobehavioral phenotype includes intellectual disability, behavioral problems, inattention and elevated rates of psychosis, the latter being first reported as more common in those with the UPD subtype of PWS compared to the deletion by Boer and colleagues (Boer et al. 2002; see also (Larson et al., 2014; Yang et al., 2013). 
Large-scale genetic association studies have identified novel schizophrenia-associated recurrent risk loci within the $15 q$ region (Tam et al., 2009), suggesting that genes within the PWS critical region may play a broader role in psychosis susceptibility in the typically developing population. Notably, a recent metaanalysis of data from five studies concluded that those with mUPD are at particularly high risk for psychosis (Yang et al., 2013). While there was no over-representation of depressive psychosis in those with mUPD, individuals with mUPD also had higher rates of bipolar illness than those with the paternal deletion. In contrast, people with PWS with paternal deletion had lower Verbal IQ scores relative to mUPD cases, suggesting that the discrepancy is not one of general syndrome severity. These findings suggest mUPD as one potential background risk factor indicating enhanced psychosis risk. However, little is known about dimensional phenotypes (i.e, prodromal symptoms) that may predict the emergence of psychosis in PWS. Nor are the behavioral correlates of such symptoms well understood.

There is evidence from polysomnographic studies of sleep disturbance in PWS, specifically sleepdisordered breathing with central apnea in very young children and excessive daytime sleepiness in school-aged and older youth (Cotton \& Richdale, 2006; Hertz et al., 1995; Vgontzas et al., 1996; Whitman \& Cataletto, 2019). However, research on the relationship of sleep disturbance to psychiatric manifestations in PWS is scarce. Sleep is a phenotype of interest in relationship to psychosis risk, given that youth at clinical high risk (CHR) for psychosis have consistently shown elevated rates of a variety of sleep disturbances (Lunsford-Avery et al., 2013, 2015; Zanini et al., 2015). These findings collectively highlight sleep disruption as not only a prominent psychiatric illness phenotype, but as a potentially important biomarker of psychosis risk.

With regard to neurocognition, global cognitive function is typically in the impaired range (average Full Scale IQ of 60, but highly variable, ranging from 40-100 across studies) (Dykens et al., 1992; Whittington \& Holland, 2017). Deficits in emotion recognition and social perception (i.e., accurately judging the intentions of others) are particularly prominent in individuals with PWS (Dimitropoulos et al., 2019; Dykens et al., 2019). There is also some evidence that cognition may differ as a function of genetic subtype: Torrado et al. found that less than 10 percent of subjects with paternal deletion had Full-Scale IQ (FSIQ) 70 or greater, whereas over $60 \%$ of subjects without the deletion subtype had FSIQ of 70 or higher (Torrado et al., 2007).

In the present study, we sought to remotely evaluate neurobehavioral traits potentially associated with psychosis risk, in order to allow for large-scale data collection; determining the feasibility of such assessments is particularly important for rare disorders, where travel to a research site may not be feasible. Specifically, we assessed dimensionally measured psychosis-risk symptoms, parent-reported sleep disturbance, and multiple domains of cognition.

\section{Methods}

Potential study participants were notified of the online study via the Foundation for Prader-Willi Research (FPWR), and through Facebook pages and other social media. In order to maximize data collection and 
study participation, our inclusion criteria were broad: 1) Genetic diagnosis of Prader-Willi Syndrome; 2) ages $12-50 ; 3$ ) able to provide informed consent or assent.

Study procedures were described in an online consent form; parents or legal guardians signed consent, and a simplified assent form was provided for participants with PWS. After consent and assent forms were completed, a link was provided to the online clinical and neurocognitive assessment forms described below. Study participants and parents each received a \$20 gift card for participation. All study procedures were approved by the UCLA Institutional Review Board.

\section{Demographic Information}

Recorded information included age, sex, race/ethnicity, personal education level, parental education level, and type of PWS mutation.

\section{Prodromal Questionnaire-Brief Version (PQ-B)}

A modified parent-report version of the Prodromal Questionnaire (PQ-B, Loewy et al., 2011) a screening measure for psychosis risk syndromes, was administered. This measure includes 21 true/false questions about unusual thoughts and experiences, and associated distress regarding these experiences ('When this happens, he/she feels frightened, concerned or it causes problems', with responses ranging from 'strongly disagree' to 'strongly agree'). It has been shown to have good concurrent validity with an interview-based diagnosis of a psychosis risk syndrome (Loewy et al., 2011).

\section{Sleep Quality}

Sleep was rated with a combination of the self-reported sleep measures, consisting of the Pittsburgh Sleep Quality Index (PSQI) (Buysse et al., 1989), the "RU-SATED" which stands for sleep regularity, satisfaction, timing, duration, efficiency, and alertness during the day (Buysse, 2014) and an additional napping frequency variable. The PSQI and the napping variables are rated on a $0-3$ scale, with higher scores indicating more severe sleep problems. Higher scores on this measure have been associated with increased positive and negative symptom severity in youth at clinical high risk for psychosis (Goines et al., 2019; Poe et al., 2017).

The RU-SATED is rated on a 0-2 scale, with higher scores indicating greater sleep disturbance. Together, the sleep measure includes: sleep duration; multiple factors related to sleep disturbance, sleep latency (i.e., the amount of time it takes to fall asleep), daytime dysfunction due to sleepiness, habitual sleep efficiency (i.e., the total time asleep divided by the amount of time in bed), subjective sleep quality, and use of sleep medication. For this study, the total sleep score was the sum of the PSQI, the napping variable, and reversed RU-SATED scores.

\section{Penn Computerized Neurocognitive Battery (Penn-CNB)}


We employed an online neurocognitive battery that has been extensively validated across a wide age range, which assesses multiple domains of cognition, specifically measures of executive function and attention, verbal and nonverbal memory, verbal and non-verbal reasoning, social cognition (emotion recognition, age differentiation), and processing speed [motor praxis; (Gur et al. 2010; Moore et al., 2015)]. The battery has been used in children with intellectual disability (Yi et al., 2016), captures both accuracy and response time, and employs automated quality assurance and scoring procedures (Moore et al., 2015).

\section{Statistical Analysis}

We first conducted an analysis of the frequency and distribution of all PQ-B symptoms reported. To test our hypothesis that the prevalence of subthreshold psychotic-like symptoms increases with age in PWS, we conducted non-parametric correlation analyses (Spearman's rho), given the (expected) non-normal distribution of the PQ-B data.

In order to test the hypothesis that mUPD patients experience increased severity of self-reported psychotic-like experiences (i.e., attenuated (prodromal) features of conceptual disorganization, perceptual anomalies and unusual thought content) we conducted analysis of covariance (ANCOVA), controlling for age, to compare patients with mUPD to those with paternal deletions and/or imprinting mutations.

Next, we aimed to identify the most informative variables relevant to psychosis prediction in PWS, by investigating inter-relationships between PQ-B symptom items endorsed, sleep measures and demographic factors (parental and personal education, age and sex). Given the many items on the original PQ-B questionnaire, one aim of the project is to develop a modified, concise version of the questionnaire, to more effectively reflect the key symptoms associated with psychosis risk in patients with PWS, but also significantly reduce participant burden. This modified questionnaire would then become a resource for future longitudinal studies in this area.

As such, the goal of our statistical approach was to identify the most informative questions/variables that can explain PQ-B distress scores (the response variables). Two methods were employed: the first was to examine the correlation coefficients for each pair of variables. A high correlation coefficient implies that the two variables are useful for predicting the other, i.e., the answer to a specific question or behavioral expression can, with a large probability, link to a PQ-B distress item. The second method is to run regression models and then find statistically significant terms, where response variables are PQ-B distress scores. An independent variable, which is significant in a regression model, means it is in a nonnegligible position explaining the response variable compared to other non-significant terms. This approach will help to narrow down the candidate list of relevant variables.

\section{Neurocognitive Abilities}

Scores on each of the individual neurocognitive measures (Face Memory, Word Memory, Attention (Continuous Performance Test), Penn Exclusion Test, Matrix Analysis, Logical Reasoning, Emotion Recognition and Age Differentiation) were transformed to z-scores based on the means and standard 
deviations from a large normative cohort, for both accuracy and speed; the Motor Praxis test assessed motor speed with no accuracy score $(\mathrm{N}=8739$; Philadelphia Neurodevelopmental Cohort; Calkins et al., 2015; Moore et al., 2015). Secondary analyses compared accuracy and speed as a function of genetic subtype. Post-hoc results were corrected for multiple comparisons $(.05 / 12$ tests, $p=.004)$. Because only 1 subject with an imprinting mutation completed neurocognitive testing this subject was excluded from secondary analyses.

\section{Data Preprocessing}

Some data transformations were conducted prior to analysis and missing values for individual questionnaire items were imputed. We used the mode of each variable, as in (Xu et al., 2020) to impute the missing values in that column. The rationale for this approach is that we assume missing values are those that have occurred most frequently because they have the highest likelihood of occurrence.

\section{Results}

Of the 128 respondents that completed demographic information, 104 additionally completed the PSQI and 98 completed the PQ-B (see participant demographics in Table 1). A subset of these participants $(n=39)$ completed the neurocognitive assessment battery.

Table 1. Participant Demographics

\begin{tabular}{|l|r|}
\hline & PWS Participants (n = 127) \\
\hline Age in years (SD) & $19.2(8.38)$ \\
\hline Age Range in years & $10-49$ \\
\hline Females, N (\%) & $68(53.5 \%)$ \\
\hline Ethnicity, N (\%) & Non-Hispanic White =110 (86.6\%) \\
\hline & Native American =1 (0.8\%) \\
\hline & African American = 3 (2.4\%) \\
\hline & Asian American = 2(1.6\%) \\
\hline & Latino/Hispanic = 7 (5.5\%) \\
\hline $\begin{array}{l}\text { Highest Parental Education in years } \\
\text { (SD) }\end{array}$ & Mixed Race/Other =4 (3.1\%) \\
\hline Genetic Subtype (\%) & Paternal Deletion $=61(48 \%)$ \\
\hline & Maternal Uniparental Disomy (UPD) $=46$ \\
& $(36.2 \%)$ \\
\hline & Imprinting = 3 (2.4\%) \\
\hline & Unknown =17 (13.4\%) \\
\hline
\end{tabular}

Frequency and age relationship with psychotic-like symptoms. Individuals with PWS endorsed a range of symptomatology (PQ-B Total Score=4.18+/-3.93; PQ-B Distress Score=14.64+/-16.46). Eleven percent $(\mathrm{N}=11)$ scored higher than the standardized clinical cut-off score (i.e., >9 points) (Fonseca-Pedrero et al., 2018). The most frequently endorsed items were those related to cognitive disorganization ('Do people 
sometimes find it hard to understand what your child is saying?' $\mathrm{N}=60 ; 61.2 \%$; 'Does your child have difficulty getting his/her point across, because they ramble or go off the track a lot when they talk?' $\mathrm{N}=50$; $51 \%$ ), suspiciousness ('Does your child feel that other people are watching him/her or talking about him/her? N=33, 33.6\%; 'Does your child find himself/herself feeling mistrustful or suspicious of other people?, $\mathrm{N}=21,21.4 \%$ ), and unusual beliefs ('Has your child felt that he/she is not in control of his/her own ideas or thoughts?, $\mathrm{N}=28,28.6 \%$; 'Has your child had the sense that some person or force is around him/her, although he/she couldn't see anyone?', $\mathrm{N}=21,21.4 \%$; 'Does your child hold beliefs that other people would find unusual or bizarre?', $\mathrm{N}=26,26.5 \%$ ). Existential fears ('Has your child ever said that he/she feels that he/she does not exist, the world does not exist, or that he/she is dead?') and visual perceptual anomalies ('Has your child seen unusual things like flashes, flames, blinding light or geometric figures?' were relatively uncommon, endorsed by $\leq 5 \%$ of the sample ( $N=2$ and 5 , respectively). Contrary to our hypothesis, however, there was no relationship between age and $\mathrm{PQ}-\mathrm{B}$ total score (Spearman's rho $=.004, p=0.97, n=95$ ) or PQ-B distress score (Spearman's rho $=.026, p=0.80, n=95$ ). Relationships remained non-significant when controlling for sex.

Relationship between PWS genetic subtype and symptomatology. There were no significant differences in $P Q-B$ total score $(F(3,91)=-.787, p=0.50)$ or distress score $(F(3,91)=1.32, p=0.27)$ as a function of $P W S$ genetic subtype (mUPD, paternal deletion, imprinting mutation or unknown). There was a trend toward a difference in PSQI total score, with the imprinting mutation associated with greater sleep disturbance $(F(3,103)=2.61, p=.055)$.

Predictors of psychotic-like symptoms- Method 1: Correlation Coefficients. Given that the majority of subjects completed the PQ-B and PSQI, our regression model focused on these measures as well as demographic variables. The first method we applied examined the linear relationships among PQ-B response variables and independent variables to determine their importance. This analysis revealed that there were no highly correlated variables. Based on a threshold correlation coefficient value of 0.4 (absolute value), only seven pairs are filtered, as shown in Table 2.

Table 2: Correlations between Predictor Items and PQ-B Distress Items

\begin{tabular}{|c|c|c|}
\hline Predictor Variable & $\begin{array}{r}\text { PQ-B Item } \\
\end{array}$ & $\begin{array}{l}\begin{array}{l}\text { Correlation } \\
\text { coefficient (r) }\end{array} \\
\end{array}$ \\
\hline PSQI Overall Sleep Quality & PQ B 1.Unusual Sounds & 0.40 \\
\hline PSQI Night-time awakening & PQ_B 3. Things appear different & 0.43 \\
\hline PSQI Daytime fatigue & $\begin{array}{r}\text { PQ_B 5. Not in control of } \\
\text { ideas/thoughts }\end{array}$ & 0.41 \\
\hline $\begin{array}{l}\text { PSQI Nightly sleep disturbance } \\
\text { (bad dreams) }\end{array}$ & $\begin{array}{r}\text { PQ_B 5. Not in control of } \\
\text { ideas/thoughts }\end{array}$ & 0.43 \\
\hline PSQI Overall Sleep Quality & $\begin{array}{r}\text { PQ_B 5. Not in control of ideas } \\
/ \text { thoughts }\end{array}$ & 0.40 \\
\hline $\begin{array}{l}\text { PSQI Nightly sleep disturbance } \\
\text { (feels cold) }\end{array}$ & $\begin{array}{r}\text { PQ_B 7. Beliefs about being } \\
\text { unusually gifted }\end{array}$ & 0.46 \\
\hline PSQI Global Sleep Disturbance & $\begin{array}{r}\text { PQ-B 18. Mistrustful or } \\
\text { suspicious }\end{array}$ & 0.44 \\
\hline
\end{tabular}


The advantage of this approach is that we can directly assess the inter-relationships between pairs of variables. However, since the variables are not highly correlated, examining correlation coefficients between each pair of variables may not be sufficient for building their association.

Method 2: Identify Variable Importance. The distributions of PQ-B distress items were found to all generally follow Poisson distributions (see Figure 1, Figure S1). Therefore, we decide to model each of them by running a Poisson regression.

Since we still have a relatively large number of candidate independent variables given the number of observations, for each model we further selected a small set of variables (i.e, a regression model with six predictors was deemed appropriate). Otherwise, we would face an overfitting problem, which means the model would not perform well on predicting future data.

Here, to implement variable selection in regression models in our dataset we applied a simulation method that is a variant of the random forest method, the goal of which is to output feature/variable importance (List et al., 2014). Specifically, the procedure of this method is as follows:

- We set a large simulation/iteration number (5000).

- At each iteration, for each response variable we build a Poisson regression model by randomly choosing 6 independent variables from the variable pool (using the same items as in Method 1 , above).

- We examine the summary statistics and find which ones are significant in each model.

- Therefore, for each response variable (PQ-B distress score), we now have 5000 Poisson models with each using a different set of independent variables chosen from the list. We summarize which independent variables are significant a greater number of times in these 5000 models. The more times a variable is significant, the more important this variable is for modelling the response variable.

- If necessary, we could continue to summarize/rank these independent variables by summing over the statistics over all response variables (all types of distress scores). Summarizing over all simulated models, the results show the importance order of independent variables (variable name, number of significant times):

As shown in Table 3, this ordering indicates that - based on our simulation method - 'Q6_all' (Reasons for sleep difficulty-Overall) appears the greatest number of times (36422), and thus is likely the most important variable explaining PQ-B distress items. The top five most important predictors/features include, in addition to 'Q6_all': morning wake time (10,878), daytime fatigue (5902), overall sleep quality (3531), 'sleep consistency_13' (awake all day without dozing/napping) (3083) and ' sleep consistency _15' (sleep 6-8 hours/night) (2194). 
Table 3. Order of Variables - Number of times appearing

Variable Name

Count

'Q6_all' (Reasons for sleep difficulty - all)

36422

'Waketime_2' (time you wake up in the morning) 10878

'FATIGUE_9' (Daytime fatigue) 5902

'PSQISLPQUAL' (Overall sleep quality) 3531

'Sleep Consistency_13' (awake all day without dozing/napping) 3083

'Sleep Consistency_15' (sleeps 6-8 hours/night) 2194

Mother's Educational Attainment - Bachelor's Degree 2162

$\begin{array}{ll}\text { Daytime Nap } & 2161\end{array}$

'Bedtime_24' (time you go to bed at night) 1864

'tmphse' (Sleep Efficiency) 1505

$\begin{array}{ll}\text { Participant Education Completed - 8th grade } & 1480\end{array}$

'Fatigue_10' (enthusiasm to get things done) 1432

$\begin{array}{ll}\text { Participant Age } & 1141\end{array}$

'newtib' (time spent asleep) 1010

'PSQIDISTB' (Overall sleep disturbance) 832

Mother's Educational Attainment - Master's Degree 825

Mother's Educational Attainment - Some college 824

$\begin{array}{lc}\text { Male Gender } & 816\end{array}$

Participant Education Completed - High school graduate $\quad 792$

$\begin{array}{ll}\text { Participant Education Completed - 6th grade } & 778\end{array}$

'PSQIDAYDYS' (Daytime dysfunction due to sleepiness) 756

Mother's Educational Attainment - Doctoral Degree 746

'HOURS' (hours of sleep per night) 684

Average nap length $\quad 665$

$\begin{array}{ll}\text { Overall Poor Sleep Quality } & 615\end{array}$

This suggests that for future iterations of the PQ-B for the PWS population, in which we wish to reduce the number of questions or indices for screening for psychosis risk, the above items should be prioritized. 


\section{Neurocognitive Domains}

Compared to the cohort of typically developing individuals, individuals with PWS showed generalized deficits in accuracy, with effect sizes ranging from moderate to large (>1SD below normative values). The deficits in accuracy were especially pronounced for tasks involving faces: namely, Face Memory and measures of social cognition involving faces (Age Differentiation and Emotion Recognition; Figure 2a). With regard to speed of performance, those with PWS performed more slowly than typically developing youth, with the greatest deficits in Attention $(z=-1.5+/-0.20)$ and Emotion Recognition $(z=-1.4+/-.21$; see Figure $2 b$ ). In secondary analyses investigating cognitive deficits as a function of mutation type (mUPD $\mathrm{N}=14$; Paternal deletion $\mathrm{N}=20$; Unknown, $\mathrm{N}=5$ ), there were no differences between groups as a function of mutation type that survived multiple comparison correction (all $p>.10$ ).

\section{Discussion}

To our knowledge, this is the first remote assessment of self-reported behavior (psychotic symptoms and sleep) and neurocognition in individuals with PWS. Results are important as they show, in a relatively large sample, a large portion of individuals with PWS experience both distressing psychotic-like experiences and disrupted sleep. The most frequently endorsed symptoms in individuals with PWS were related to cognitive disorganization, endorsed in about half the sample, and suspiciousness, endorsed in about $25 \%$ of the sample. Regarding relationships between sleep disturbance, as measured by the PSQI, and psychotic-like symptoms, as assessed by the PQ-B, both of the methods we employed revealed that questions on sleep behavior/habits and fatigue are relatively important for explaining distress related to unusual experiences and other psychotic-like symptomatology. While excessive daytime sleepiness is a common, well -established phenomenon in individuals with PWS, related to hypothalamic dysfunction (Salles et al., 2020), our findings also show it is associated with psychotic-like symptoms. While we cannot determine cause and effect in this cross-sectional study, these findings nevertheless suggest that disrupted sleep may be a precursor to psychotic symptomatology. As a potentially modifiable risk factor, this offers important new information. Other demographic variables, such as age, sex, and personal and parental education level were not as strongly associated with psychosis risk.

This novel web-based approach offers substantial cost savings and efficiency, as no subject travel was required. Further, it allows families in rural locations to participate, who are otherwise unlikely to be able to take part in this research. The development of more scalable methods for research participation is important not only for rare disorders, but for other populations that lack adequate access, such as families with limited financial resources (Hyde et al., 2020).

In help-seeking youth in the general population, the PQ-B shows good concurrent validity with interviewbased diagnoses of a psychosis risk syndrome (Loewy et al., 2011). However, certain highly endorsed PQ$B$ items in individuals with PWS may be related to global cognitive dysfunction, and thus may not have the same prognostic significance. Longitudinal studies are warranted to evaluate predictive validity of individual PQ-B items in the PWS population. 
Contrary to expectations based on prior reports of higher rates of overt psychotic disorder in PWS resulting from mUPD (Boer et al. 2002; Soni et al. 2008), we did not see a difference in parent-reported psychotic-like symptomatology as a function of PWS genetic subtype. However, there are a number of reasons this may be the case. First, psychotic-like experiences are not equivalent to a psychotic disorder, and prior evidence suggests that psychotic symptoms in individuals with PWS have an uncharacteristically acute onset (Aman et al., 2018;)Holland et al., 2003). The phenomenology of psychosis in PWS also is distinct from 'typical' psychosis presentation, in that there is a strong affective component and a more 'cycloid' pattern (Larson et al. 2014). As such, further modifications to our questionnaire based on retrospective studies may be needed to better address these components.

Although our cognitive assessment was only completed by a subset of the larger sample, findings reveal differential impairment in social cognitive domains, particularly tasks involving processing of faces, implicating cortico-limbic dysfunction (Gur et al., 2017). This pattern of findings is highly consistent with other recent work in individuals with PWS, involving in-lab assessment (Dykens et al. 2019; Debladis et al., 2019). Another recent study offers a translational perspective on psychiatric manifestations of PWS, through the RDoC matrix (Salles et al., 2020): deficits in social processes are highlighted, with eventrelated potential (ERP) evidence that PWS patients have altered processing of faces (Key et al., 2013). It has been hypothesized that dysfunctional hypothalamic oxytocin-expressing neurons may underlie these deficits in PWS (Swaab et al., 1995).

\section{Limitations}

Several limitations of the current study should be noted. First, this may not be a representative sample of people with PWS; for example, those who may have been mentally unwell at the time of the study are unlikely to have taken part. Secondly, the online format of the study limited the amount of detailed prior history we could obtain on study participants. As such, we were only able to obtain cross-sectional information on current (past month) sleep and psychotic-like experiences. Thirdly, we do not have information on the use of psychiatric medications, which -if being used - might suppress symptoms. Finally. we also had a much more limited number of completers for the neurocognitive assessment (relative to questionnaires), and thus the neurocognitive performance variables could not be included in our predictive model.

\section{Conclusions}

PWS is associated with a high prevalence of distressing psychotic-like experiences, which were associated with sleep disturbance. Social cognition, particularly tasks involving faces, showed differential impairment. These results support feasibility of remote assessment of individuals with rare neurogenetic syndromes.

\section{Declarations}


Ethics approval and consent to participate: All study procedures were approved by the UCLA Institutional Review Board. Participants with PWS and their parents electronically signed informed assent/consent documents, respectively.

Consent for publication: Not applicable

Availability of data and materials: The deidentified dataset supporting the conclusions of this article is available from the corresponding author on reasonable request.

Competing Interests/Disclosures: The authors have no relevant financial disclosures or conflicts of interest.

Authors' contributions: CEB designed the study and drafted the manuscript. ZZ conducted statistical analyses and drafted methods and results text. $Z Z, Z H$, and $A V$ contributed to data management, quality control and analyses. LP-H, LK-W, and AV oversaw recruitment and participant assessment. AMP and RCG designed the neurocognitive battery and provided critical input on interpretation of results. ER and $\mathrm{AH}$ provided critical input on the manuscript text and interpretation of results. All authors participated in manuscript revisions and have given final approval of this version for submission.

Acknowledgements: We are grateful to the participants and their families for contributing to this work. We also thank Lauren Roth, Jessica Bohonowych, and Theresa Strong at the FWPR for their guidance, and assistance in publicizing the study.

Authors' information: The authors have expertise in the study of rare neurogenetic disorders and psychosis.

\section{References}

Aman, L. C. S., Manning, K. E., Whittington, J. E., \& Holland, A. J. (2018). Mechanistic insights into the genetics of affective psychosis from Prader-Willi syndrome. The Lancet. Psychiatry, 5(4), 370-378. https://doi.org/10.1016/S2215-0366(18)30009-9

Boer, H., Holland, A.J., Whittington, J., Butler, J., Webb, T. and Clarke, D. (2002). Psychotic illness in people with Prader-Willi Syndrome due to chromosome 15 maternal uniparental disomy The Lancet, 359: 135-136.

Buysse, D. J. (2014). Sleep health: Can we define it? Does it matter? Sleep, 37(1), 9-17. https://doi.org/10.5665/sleep.3298

Buysse, D. J., Reynolds, C. F., Monk, T. H., Berman, S. R., \& Kupfer, D. J. (1989). The Pittsburgh sleep quality index: A new instrument for psychiatric practice and research. Psychiatry Research, 28(2), 193213. https://doi.org/10.1016/0165-1781(89)90047-4 
Calkins, M. E., Merikangas, K. R., Moore, T. M., Burstein, M., Behr, M. A., Satterthwaite, T. D., Ruparel, K., Wolf, D. H., Roalf, D. R., Mentch, F. D., Qiu, H., Chiavacci, R., Connolly, J. J., Sleiman, P. M. A., Gur, R. C., Hakonarson, H., \& Gur, R. E. (2015). The Philadelphia Neurodevelopmental Cohort: Constructing a deep phenotyping collaborative. Journal of Child Psychology and Psychiatry, and Allied Disciplines, 56(12), 1356-1369. https://doi.org/10.1111/jcpp.12416

Cassidy, S. B., Schwartz, S., Miller, J. L., \& Driscoll, D. J. (2012). Prader-Willi syndrome. Genetics in Medicine, 14(1), 10-26. https://doi.org/10.1038/gim.0b013e31822bead0

Cotton, S., \& Richdale, A. (2006). Brief report: Parental descriptions of sleep problems in children with autism, Down syndrome, and Prader-Willi syndrome. Research in Developmental Disabilities, 27(2), 151161. https://doi.org/10.1016/j.ridd.2004.12.003

Debladis, J., Valette, M., Strenilkov, K. Mantoulan, Thuilleaux, Laurier, Molinas, Pascal Barone and Tauber, M. Face processing and exploration of social signals in Prader-Willi syndrome: a genetic signature. Orphanet J Rare Dis 14, 262 (2019). https://doi.org/10.1186/s13023-019-1221-3

Dimitropoulos, A., Zyga, O., \& Russ, S. W. (2019). Early Social Cognitive Ability in Preschoolers with Prader-Willi Syndrome and Autism Spectrum Disorder. Journal of Autism and Developmental Disorders, 49(11), 4441-4454. https://doi.org/10.1007/s10803-019-04152-4

Dykens, Elisabeth M., Hodapp, R. M., Walsh, K., \& Nash, L. J. (1992). Profiles, Correlates, and Trajectories of Intelligence in Prader-Willi Syndrome. Journal of the American Academy of Child \& Adolescent Psychiatry, 31(6), 1125-1130. https://doi.org/10.1097/00004583-199211000-00022

Dykens, Elisabeth M., Roof, E., Hunt-Hawkins, H., Daniell, C., \& Jurgensmeyer, S. (2019). Profiles and trajectories of impaired social cognition in people with Prader-Willi syndrome. PLOS ONE, 14(10), e0223162. https://doi.org/10.1371/journal.pone.0223162

Fonseca-Pedrero, E., Inchausti, F., Pérez-Albéniz, A., \& Ortuño-Sierra, J. (2018). Validation of the Prodromal Questionnaire-Brief in a representative sample of adolescents: Internal structure, norms, reliability, and links with psychopathology. International Journal of Methods in Psychiatric Research, 27(4), e1740. https://doi.org/10.1002/mpr.1740

Goines, K. B., LoPilato, A. M., Addington, J., Bearden, C. E., Cadenhead, K. S., Cannon, T. D., Cornblatt, B. A., Mathalon, D. H., McGlashan, T. H., Perkins, D. O., Tsuang, M. T., Woods, S. W., \& Walker, E. F. (2019). Sleep problems and attenuated psychotic symptoms in youth at clinical high-risk for psychosis. Psychiatry Research, 282, 112492. https://doi.org/10.1016/j.psychres.2019.112492

Gur RC, Richard J, Hughett P, Calkins ME, Macy L, Bilker WB, Brensinger C, Gur RE. A cognitive neuroscience-based computerized battery for efficient measurement of individual differences: standardization and initial construct validation. J Neurosci Methods. 2010 Mar 30;187(2):254-62. doi: 10.1016/j.jneumeth.2009.11.017. Epub 2009 Nov 27. PMID: 19945485; PMCID: PMC2832711. 
Gur, R. E., Moore, T. M., Calkins, M. E., Ruparel, K., \& Gur, R. C. (2017). Face Processing Measures of Social Cognition: A Dimensional Approach to Developmental Psychopathology. Biological Psychiatry. Cognitive Neuroscience and Neuroimaging, 2(6), 502-509. https://doi.org/10.1016/j.bpsc.2017.03.010

Hertz, G., Cataletto, M., Feinsilver, S. H., \& Angulo, M. (1995). Developmental trends of sleep-disordered breathing in Prader-Willi syndrome: The role of obesity. American Journal of Medical Genetics, 56(2), 188-190. https://doi.org/10.1002/ajmg.1320560215

Holland, A., Whittington, J., \& Hinton, E. (2003). The paradox of Prader-Willi syndrome: A genetic model of starvation. The Lancet, 362(9388), 989-991. https://doi.org/10.1016/S0140-6736(03)14370-X

Hyde C, Pizzano M, McDonald NM, Nelson CA 3rd, Kasari C, Thiele EA, Jeste SS. A telehealth approach to improving clinical trial access for infants with tuberous sclerosis complex. J Neurodev Disord. 2020 Jan 22;12(1):3. doi: 10.1186/s11689-019-9302-0. PMID: 31969108; PMCID: PMC6977334.

Key, A. P., Jones, D., \& Dykens, E. M. (2013). Social and emotional processing in Prader-Willi syndrome: Genetic subtype differences. Journal of Neurodevelopmental Disorders, 5(1), 7.

https://doi.org/10.1186/1866-1955-5-7

Larson, F. V., Whittington, J., Webb, T., \& Holland, A. J. (2014). A longitudinal follow-up study of people with Prader-Willi syndrome with psychosis and those at increased risk of developing psychosis due to genetic subtype. Psychological Medicine, 44(11), 2431-2435.

https://doi.org/10.1017/S0033291713002961

List, M., Hauschild, A.-C., Tan, Q., Kruse, T. A., Baumbach, J., \& Batra, R. (2014). Classification of Breast Cancer Subtypes by combining Gene Expression and DNA Methylation Data. Journal of Integrative Bioinformatics, 11(2), 1-14. https://doi.org/10.1515/jib-2014-236

Loewy, R. L., Pearson, R., Vinogradov, S., Bearden, C. E., \& Cannon, T. D. (2011). Psychosis risk screening with the Prodromal Questionnaire-Brief version (PQ-B). Schizophrenia Research, 129(1), 42-46. https://doi.org/10.1016/j.schres.2011.03.029

Lunsford-Avery, J. R., LeBourgeois, M. K., Gupta, T., \& Mittal, V. A. (2015). Actigraphic-measured sleep disturbance predicts increased positive symptoms in adolescents at ultra high-risk for psychosis: $A$ longitudinal study. Schizophrenia Research, 164(1), 15-20. https://doi.org/10.1016/j.schres.2015.03.013

Lunsford-Avery, J. R., Orr, J. M., Gupta, T., Pelletier-Baldelli, A., Dean, D. J., Smith Watts, A. K., Bernard, J., Millman, Z. B., \& Mittal, V. A. (2013). Sleep dysfunction and thalamic abnormalities in adolescents at ultra high-risk for psychosis. Schizophrenia Research, 151(1), 148-153.

https://doi.org/10.1016/j.schres.2013.09.015

Moore, T. M., Reise, S. P., Gur, R. E., Hakonarson, H., \& Gur, R. C. (2015). Psychometric properties of the Penn Computerized Neurocognitive Battery. Neuropsychology, 29(2), 235-246. 
Poe, S.-L., Brucato, G., Bruno, N., Arndt, L. Y., Ben-David, S., Gill, K. E., Colibazzi, T., Kantrowitz, J. T., Corcoran, C. M., \& Girgis, R. R. (2017). Sleep disturbances in individuals at clinical high risk for psychosis. Psychiatry Research, 249, 240-243. https://doi.org/10.1016/j.psychres.2016.12.029

Salles, J., Lacassagne, E., Benvegnu, G., Berthoumieu, S. Ç., Franchitto, N., \& Tauber, M. (2020). The RDoC approach for translational psychiatry: Could a genetic disorder with psychiatric symptoms help fill the matrix? the example of Prader-Willi syndrome. Translational Psychiatry, 10. https://doi.org/10.1038/s41398-020-00964-6

Swaab, D. F., Purba, J. S., \& Hofman, M. A. (1995). Alterations in the hypothalamic paraventricular nucleus and its oxytocin neurons (putative satiety cells) in Prader-Willi syndrome: A study of five cases. The Journal of Clinical Endocrinology and Metabolism, 80(2), 573-579.

https://doi.org/10.1210/jcem.80.2.7852523

Tam, G. W. C., Redon, R., Carter, N. P., \& Grant, S. G. N. (2009). The role of DNA copy number variation in schizophrenia. Biological Psychiatry, 66(11), 1005-1012.

https://doi.org/10.1016/j.biopsych.2009.07.027

Torrado, M., Araoz, V., Baialardo, E., Abraldes, K., Mazza, C., Krochik, G., Ozuna, B., Leske, V., Caino, S., Fano, V., \& Chertkoff, L. (2007). Clinical-etiologic correlation in children with Prader-Willi syndrome (PWS): An interdisciplinary study. American Journal of Medical Genetics Part A, 143A(5), 460-468. https://doi.org/10.1002/ajmg.a.31520

Vgontzas, A. N., Kales, A., Seip, J., Mascari, M. J., Bixler, E. O., Myers, D. C., Vela-Bueno, A., \& Rogan, P. K. (1996). Relationship of sleep abnormalities to patient genotypes in Prader-Willi syndrome. American Journal of Medical Genetics, 67(5), 478-482. https://doi.org/10.1002/(SICl)10968628(19960920)67:5<478::AID-AJMG7>3.0.C0;2-G

Webb, T., Whittington, J., Clarke, D., Boer, H., Butler, J., \& Holland, A. (2002). A study of the influence of different genotypes on the physical and behavioral phenotypes of children and adults ascertained clinically as having PWS. Clinical Genetics, 62(4), 273-281. https://doi.org/10.1034/j.13990004.2002.620404.x

Whitman, B. Y., \& Cataletto, M. E. (2019). Prader-Willi Syndrome. In J. A. Accardo (Ed.), Sleep in Children with Neurodevelopmental Disabilities: An Evidence-Based Guide (pp. 195-201). Springer International Publishing. https://doi.org/10.1007/978-3-319-98414-8_16

Whittington, J., \& Holland, A. (2017). Cognition in people with Prader-Willi syndrome: Insights into genetic influences on cognitive and social development. Neuroscience \& Biobehavioral Reviews, 72, 153-167. https://doi.org/10.1016/j.neubiorev.2016.09.013 
Xu, X., Xia, L., Zhang, Q., Wu, S., Wu, M., \& Liu, H. (2020). The ability of different imputation methods for missing values in mental measurement questionnaires. BMC Medical Research Methodology, 20(1), 42. https://doi.org/10.1186/s12874-020-00932-0

Yang, L., Zhan, G., Ding, J., Wang, H., Ma, D., Huang, G., \& Zhou, W. (2013). Psychiatric illness and intellectual disability in the Prader-Willi syndrome with different molecular defects-A meta analysis. PloS One, 8(8), e72640. https://doi.org/10.1371/journal.pone.0072640

Yi, J. J., Weinberger, R., Moore, T. M., Calkins, M. E., Guri, Y., McDonald-McGinn, D. M., Zackai, E. H., Emanuel, B. S., Gur, R. E., Gothelf, D., \& Gur, R. C. (2016). Performance on a computerized neurocognitive battery in 22q11.2 deletion syndrome: A comparison between US and Israeli cohorts. Brain and Cognition, 106, 33-41. https://doi.org/10.1016/j.bandc.2016.02.002

Zanini, M. A., Castro, J., Cunha, G. R., Asevedo, E., Pan, P. M., Bittencourt, L., Coelho, F. M., Tufik, S., Gadelha, A., Bressan, R. A., \& Brietzke, E. (2015). Abnormalities in sleep patterns in individuals at risk for psychosis and bipolar disorder. Schizophrenia Research, 169(1), 262-267.

https://doi.org/10.1016/j.schres.2015.08.023

\section{Figures}



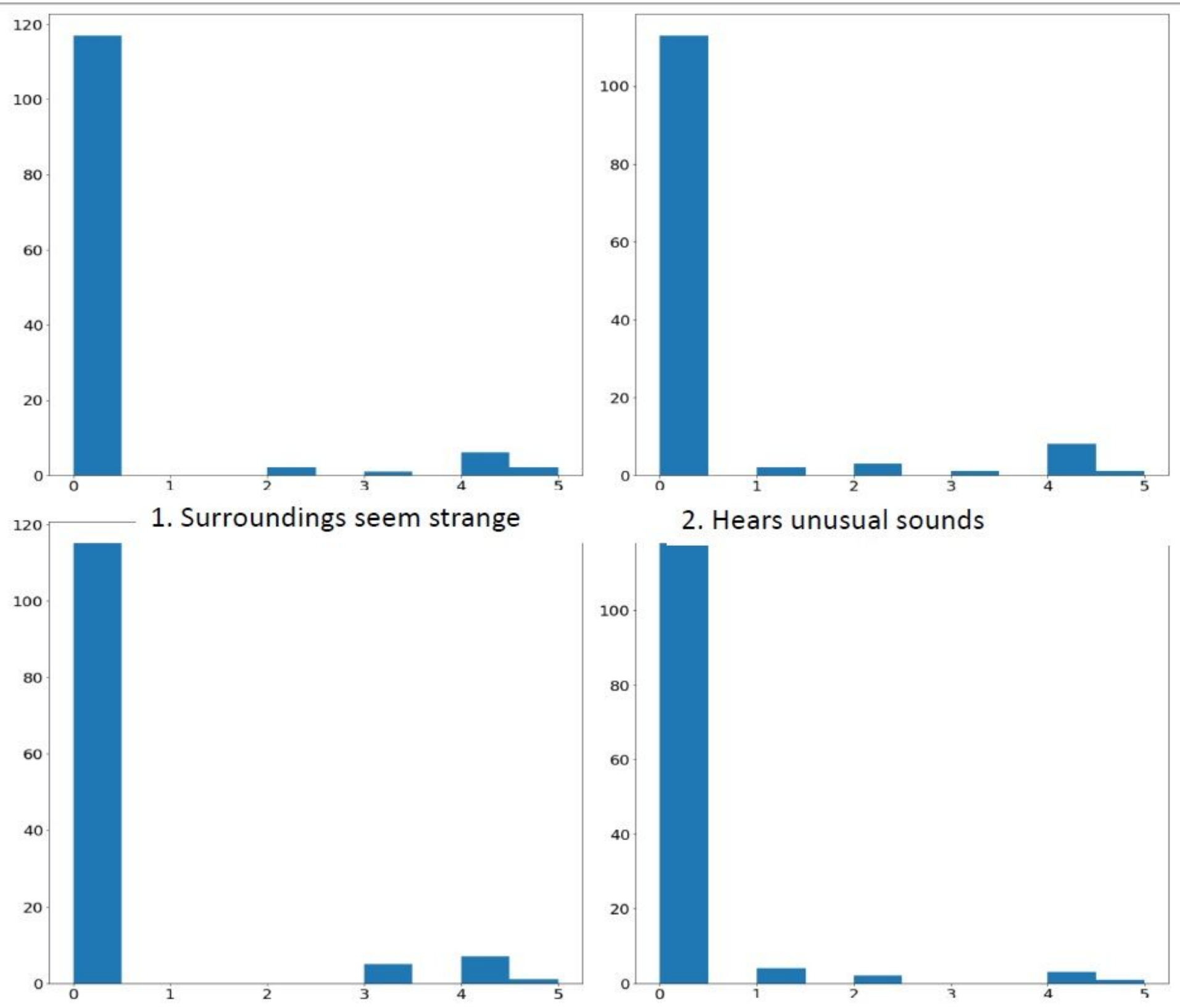

3. Visual- 'things appear different'

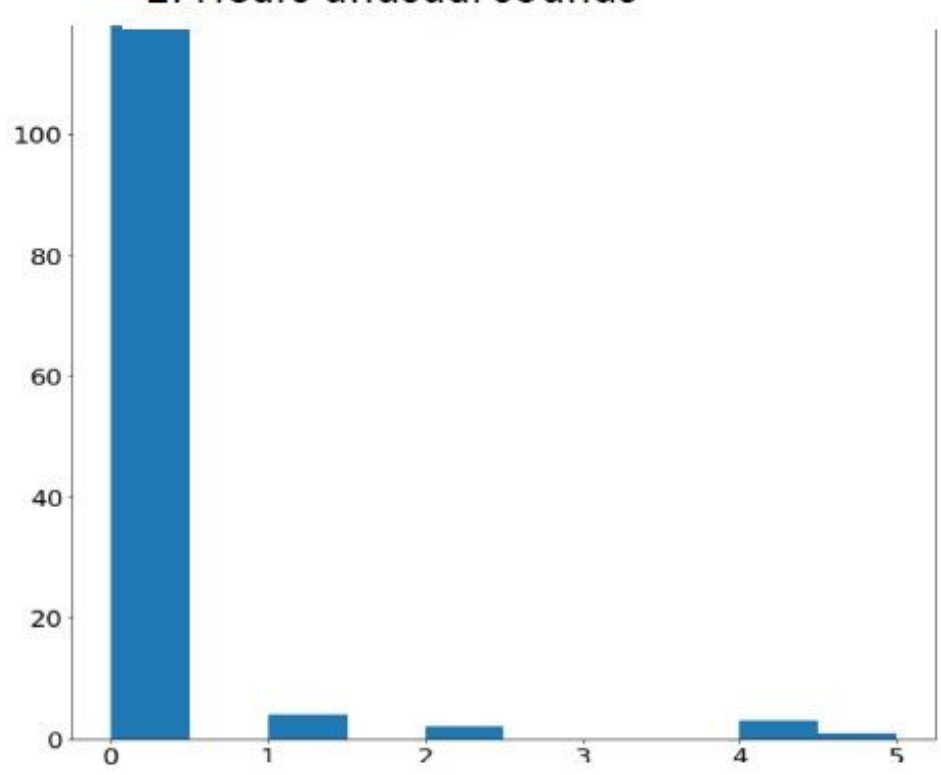

4. Telepathy/psychic forces

\section{Figure 1}

Distribution of PQ-B Distress variables. All variables were found to follow a Poisson distribution. Four variables are randomly shown for ease of visualization; the other response variables have a similar distribution pattern (see Supplementary Figure 1). 

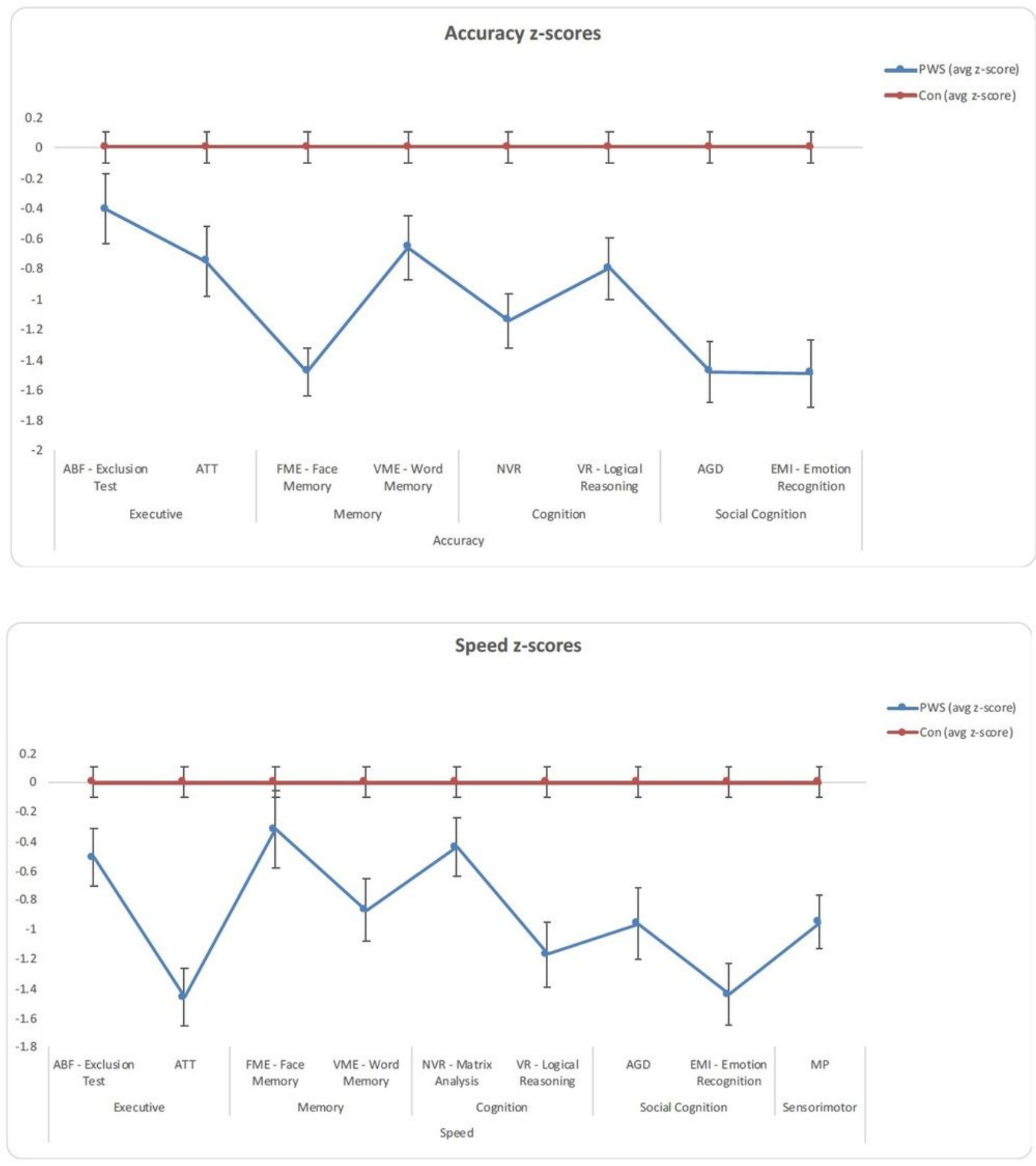

\section{Figure 2}

a. Penn-CNB Results: Average accuracy z-scores across domains in PWS vs. typically developing cohort (Calkins et al., 2015). ABF-Exclusion= Penn Exclusion Test, ATT=Attention (Continuous Performance Test), AGD=Age Differentiation. Individuals with PWS showed greatest deficits in the domains of Face Memory and Social Cognition (Age Differentiation and Emotion Recognition). b. Penn-CNB Results: Average speed z-scores across domains in PWS vs. typically developing cohort. Abbreviations same as 
above, plus MP= Motor Praxis. Individuals with PWS showed greatest deficits (slowest speed) in the domains of Attention and Emotion Recognition.

\section{Supplementary Files}

This is a list of supplementary files associated with this preprint. Click to download.

- SupplementaryFigure1.pdf 